\title{
Vertikale Blickparesen bei dorsalem Mittelhirnsyndrom und anderen Erkrankungen
}

\author{
Berthold Pemp (D) - Sabine Koinig · Karl Kircher · Christoph Mitsch · Andreas Reitner
}

Eingegangen: 4. Mai 2020 / Angenommen: 6. Januar 2021 / Online publiziert: 13. Januar 2021

(C) Der/die Autor(en) 2021

\begin{abstract}
Zusammenfassung
Hintergrund Die vertikale Blickparese (vBP) ist ein typisches Symptom bei Läsionen des dorsalen Mittelhirns. Die klinische Ausprägung der vBP kann dabei stark variieren und im Rahmen eines dorsalen Mittelhirnsyndroms (DMS) mit zusätzlichen Symptomen wie einer Konvergenzparese, einem Konvergenzretraktionsnystagmus, einer verminderten Pupillenreaktion oder einer Skew Deviation vergesellschaftet sein. Je nach Lokalisation und Größe der Läsion kann auch eine Kombination mit weiteren zentralen Motilitätsstörungen vorliegen.

Material und Methode Im Rahmen einer retrospektiven Studie wurden die Patientendaten unserer neuroophthalmologischen Ambulanz über einen Zeitraum von 25 Jahren nach Fällen mit Einschränkungen konjugierter vertikaler Augenbewegungen durchsucht und hinsichtlich ihrer neuroorthoptischen Befunde und Ätiologien ausgewertet.

Resultate Es wurden 202 Patienten mit vBP identifiziert. Das Befundspektrum reichte von einer isolierten Sakkadenstörung nur nach oben bis hin zum kompletten Ausfall aller willkürlichen und reflektorischen vertikalen Augenbewegungen. Nur 12 vBP lagen isoliert vor. Ursächlich war bei 155 Patienten ein DMS, wovon aber nur 18 Fälle dem von Parinaud geprägten Syn-
\end{abstract}

Assoc. Prof. PD Dr. B. Pemp ( $₫) \cdot$ K. Kircher · C. Mitsch • A. Reitner

Universitätsklinik für Augenheilkunde und Optometrie, Neuroophthalmologische Ambulanz, Medizinische Universität Wien, Währinger Gürtel 18-20, 1090 Wien, Österreich

berthold.pemp@meduniwien.ac.at

S. Koinig

Universitätsklinik für Augenheilkunde und Optometrie, Neuroophthalmologische Ambulanz, Universitätsklinikum AKH Wien, Währinger Gürtel 18-20, 1090 Wien, Österreich drombild (vBP + Konvergenzparese \pm Pupillenstörung ohne weitere Symptome) entsprachen; 42 Patienten mit DMS zeigten lediglich eine Sakkadenparese. Der Aufblick war bei DMS insgesamt mehr eingeschränkt als der Abblick. Die häufigsten Begleitsymptome waren eine Konvergenzparese ( $49 \%$ ), Konvergenznystagmus (40\%), Pupillenstörung (32\%), Skew Deviation (21\%) und Blickrichtungsnystagmus (15\%). In 58 Fällen traten weitere zentrale Okulomotorikstörungen auf. Bis zum 18. Lebensjahr war eine vBP bei $58 \%$ der Patienten durch Gehirntumoren bedingt, wohingegen nach dem 60. Lebensjahr neben einem DMS durch Infarkte (41\%) v. a. degenerative Erkrankungen (44\%) ursächlich waren.

Schlussfolgerungen Langsame und schnelle Augenbewegungen nach oben und unten werden im Hirnstamm unterschiedlich generiert, geleitet und integriert und können daher bei Läsionen des Mittelhirns verschieden stark gestört sein. Die Ergebnisse verdeutlichen zudem die enge Lagebeziehung der Zentren für vertikale Augenbewegungen, Konvergenz und Pupillenreaktion im dorsalen Mittelhirn. Die Kombination einer vBP mit weiteren neuroophthalmologischen Diagnosen kann daher eine topografische und ätiologische Zuordnung erleichtern.

Schlüsselwörter Vertikale Blickparese .

Sakkadenparese - Dorsales Mittelhirnsyndrom . Parinaud-Syndrom · Progressive supranukleäre Blickparese

\section{Vertical gaze palsies in dorsal midbrain syndrome and other diseases}

\section{Summary}

Background Vertical gaze palsy (vGP) is a typical symptom in lesions of the dorsal midbrain. The clinical picture of vGP is variable and can be part 
of a dorsal midbrain syndrome (DMS) in combination with additional symptoms including convergence paresis, convergence-retraction nystagmus, disturbed pupil reaction or skew deviation. Depending on the size and site of the lesion other central oculomotor disorders can also be present.

Material and methods The patient files of the neuroophthalmology clinic over a period of 25 years were reviewed for cases with conjugated vertical oculomotor disorders. The records were retrospectively analyzed concerning the neuro-orthoptic findings and etiologies.

Results A total of 202 patients with vGP were identified. There was a continuous spectrum of findings from isolated paresis of upward saccades to a complete loss of all voluntary and reflectory vertical eye movements. Only 12 vGP were clinically isolated. There were 155 patients with a DMS but only 18 corresponded to the clinical picture as characterized by Parinaud (vGP + convergence paresis \pm pupil disturbance without further symptoms). Of the patients with DMS 42 showed only a paresis of vertical saccades. In DMS the upgaze was generally more impaired than the downgaze. The most frequently associated symptoms were convergence paresis (49\%), convergence nystagmus (40\%), pupil disturbance (32\%), skew deviation (21\%) and gaze-evoked nystagmus $(15 \%)$. A total of 58 cases had further central oculomotor disorders. In patients up to 18 years of age vGP was caused by cerebral tumors in $58 \%$, whereas above the age of 60 years degenerative diseases (44\%) and DMS after infarcts (41\%) were the predominant causes.

Conclusion Slow and fast eye movements of upgaze and downgaze are differently generated, conducted and integrated into the brainstem and can be variably affected in midbrain lesions. The findings also illustrate the proximity of centers responsible for vertical eye movements, convergence and pupil reaction in the dorsal midbrain. The combination of a vGP with other neuro-ophthalmologic diagnoses can therefore help in topographical and etiological allocation.

Keywords Vertical gaze palsy · Saccadic paresis . Dorsal midbrain syndrome - Parinaud syndrome . Progressive supranuclear palsy

\section{Hintergrund}

Es ist faszinierend, wie komplex die Steuerung von etwas scheinbar so Einfachem wie den Augenbewegungen im Gehirn organisiert ist. Die dabei involvierten Regionen umfassen sowohl mehrere kortikale Areale, gut lokalisierbare Kerne und Schaltstellen im Hirnstamm sowie Regelkreise mit Kleinhirn und Innenohr. Dabei sind für die verschiedenen Modalitäten von schnellen und langsamen Augenbewegungen verschiedene Steuerungsbahnen angelegt, auch wenn sie in die gleiche Richtung gehen $[1,2]$. So laufen die
Bahnen für langsame Folgebewegung, Sakkaden und vestibulookulären Reflex (VOR) bis zu den schlussendlich ausführenden Hirnnervenkernen im Hirnstamm zu einem großen Teil voneinander getrennt. Zusätzlich kommt es erst durch das Zusammenspiel von erregenden und hemmenden pränukleären Bahnen, zwischengeschalteten Integratorzentren und internukleärer Verschaltung im Hirnstamm zu einer exakten und nur teilweise bewusst wahrgenommenen konjugierten Ausrichtung der Augen in der den jeweiligen bewegungsauslösenden Reizen genau angepassten Geschwindigkeit und Amplitude.

Während die finalen Schaltzentren für horizontale Augenbewegungen großteils im unteren Hirnstamm lokalisiert sind, konjugieren die Steuerungsbahnen für vertikale Augenbewegungen letztendlich im oberen Hirnstamm. Diese räumliche Trennung der Zentren für horizontale und vertikale Augenbewegungen hat zur Folge, dass pontine Läsionen vorwiegend zu Störungen der horizontalen Augenbewegungen führen (horizontale Blickparese, Abduzensparese, internukleäre Ophthalmoplegie etc.), während Pathologien im Mittelhirn eher die vertikalen Augenbewegungen beeinträchtigen.

Die vertikale Blickparese (vBP) ist dabei ein typisches Symptom bei Läsionen des dorsalen Mittelhirns. Da, wie schon erwähnt, die verschiedenen Bewegungsmodalitäten bis kurz vor dem Effektorkern des N. oculomotorius (nN.III) unterschiedlich angesteuert werden, kann die klinische Ausprägung einer vBP in der orthoptischen Untersuchung stark variieren und unter Umständen nur einzelne Bewegungsarten betreffen. Für das Verständnis dieser Dissoziation

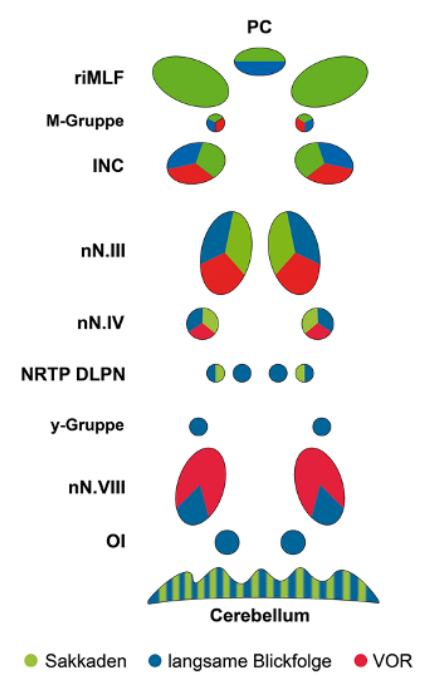

Abb. 1 Schematische Darstellung der neuroanatomischen Strukturen für die Generierung der verschiedenen Modi vertikaler Augenbewegungen im Hirnstamm. (Nach [1, 2]. PC posteriore Kommissur, riMLF rostraler interstitieller Kern des medialen Längsbündels, INC interstitieller Nucleus Cajal, $n N$.III Okulomotoriuskern, $n N$.IV Trochleariskern, NRTP Nucleus reticularis tegmenti pontis, DLPN dorsolateraler pontiner Nucleus, $n N$.VIII Vestibulariskern, OI Oliva inferior) 
und der Assoziation mit weiteren charakteristischen okulären Symptomen ist eine genauere Betrachtung der neuronalen Organisation im Hirnstamm hilfreich (s. auch Abb. 1): Die vertikale langsame Folgebewegung wird nach kortikaler Erregung in pontinen Kernen generiert (rostraler Nucleus reticularis tegmenti pontis, dorsolateraler pontiner Nucleus) und läuft über mehrere Zwischenstationen in Hirnstamm und Kleinhirn zum Hauptintegratorkern für vertikale Augenbewegungen, dem interstitiellen Nucleus Cajal (INC) und zum nN.III. Die vertikalen Sakkaden werden kortikal ähnlich gebahnt, unter Kontrolle von Kleinhirn und pontinen Strukturen jedoch im rostralen Mittelhirn generiert (rostraler interstitieller Kern des medialen Längsbündels [riMLF]). Beim Aufblick werden durch mehrfache kreuzende Projektion die INC und nN.III (rectus superior und obliquus inferior Subnuclei) beider Seiten erregt. Beim Abblick erfolgt jedoch eine vorwiegend ipsilaterale Erregung des nN.III (rectus inferior Subnucleus) und des Trochleariskerns (nN.IV). Da normalerweise eine bilaterale Stimulation vorliegt, ergibt sich trotzdem eine konjugierte Abwärtsbewegung beider Augen. Der vestibulookuläre Reflex (VOR) hat als schnellstmögliche Augenbewegung die wenigsten Synapsen und führt bei Stimulation des anterioren Bogengangs durch Generation im oberen Vestibulariskern der Medulla oblongata $\mathrm{zu}$ kontralateraler Erregung in INC und $\mathrm{nN}$.III (rectus superior und obliquus inferior Subnuclei) und bei Stimulation des posterioren Bogengangs durch Generation im medialen Vestibulariskern zur kontralateralen Erregung in INC, nN.III (rectus inferior Subnucleus) und nN.IV. Da der rectus superior Subnucleus den kontralateralen Muskel innerviert, ergäben sich durch die beschriebenen Projektionen bei einseitiger Erregung immer Augenbewegungen mit gleichsinniger vertikaler und torsioneller Komponente an beiden Augen: gleichzeitige Aktivierung von M. obliquus inferior und kontralateralem $\mathrm{M}$. rectus superior bzw. von M. rectus inferior und kontralateralem M. obliquus superior. Allerdings kommt es auch beim VOR normalerweise immer zu einer beidseitigen Erregung: Bei Kopfbewegungen in der Nick-Ebene werden durch die schräge Anordnung der Bogengänge im Raum nur die entsprechenden vertikalen VORKomponenten aktiviert, welche dann zu einer gegensinnigen konjugierten Vertikalbewegung beider Augen führen.

Der bilateral angelegte INC in unmittelbarer Nachbarschaft zum nN.III führt somit bei allen Modi der vertikalen Augenbewegungen eine immens wichtige Funktion als Integrator für erregende und hemmende Signale aus und dient dabei v. a. der Blickhaltefunktion, trägt aber auch zur Sakkadengenerierung, Antagonistenhemmung und Koordination des VOR in der Roll-Ebene bei. Ein großer Teil seiner Fasern wird über die posteriore Kommissur (PC) mit dem INC der Gegenseite ausgetauscht. Diese Fasern sind v. a. für die Regulation des Aufblicks zuständig, und zusätzlich enthält die PC noch einen eigenen Kern als Aufblickintegrator. Schädigungen in der PC führen daher vorwiegend zu einer Störung des Aufblicks. Als Hauptintegratoren der vertikalen Augenbewegungen projizieren die INCs zu den nN.III und nN.IV beider Seiten sowie ipsilateral zu riMLF, pontinem paramedianem Trakt und Vestibulariskern. Zwischen dem vertikalen Sakkadengenerator riMLF und dem Integrator INC befindet sich rostral des nN.III in der supraokulomotorischen Area (SOA) mit der M-Gruppe noch eine weitere Struktur mit Heberwirkung. Diese hat kurze Projektionen auf die Augen- und Lidheberanteile des nN.III (rectus superior und obliquus superior Subnuclei und unpaarer Nucleus caudalis centralis) und lange Projektionen auf den Fazialiskern (M. frontalis) in der Pons. In der SOA befinden sich auch noch dorsal des nN.III, von diesem aber getrennt, die parasympathischen Neurone des N.III für Pupillenverengung und Akkommodation, welche von in der PC teilweise kreuzenden Fasern der prätektalen Nuclei angesteuert werden, sowie dazwischen locker verteilt die Konvergenz steuernden Neurone [3]. Ventral des nN.III verlaufen im medialen Längsbündel noch die aufsteigenden prä- und internukleären Neuronen für vertikale und torsionale Augenbewegungen.

Somit sind auf engem Raum um die Kerngebiete für vertikale Augenbewegungen weitere Zentren und Fasern für definierte okuläre Funktionen gruppiert. Läsionen im dorsalen Mittelhirn werden daher häufig nicht nur durch Störung der vertikalen Augenbewegungen, sondern in Kombination mit entsprechenden Funktionsausfällen der Pupillenreaktion und Konvergenz symptomatisch.

\section{Material und Methode}

Im Rahmen der vorliegenden Studie wurden die Ausprägungen der vBP, ihre Assoziation mit weiteren okulären Symptomen und ätiologische Charakteristika in bestimmten Patientengruppen untersucht. Dazu wurden sämtliche Patientendaten der neuroophthalmologischen Ambulanz der Universitätsklinik für Augenheilkunde und Optometrie am AKH Wien über einen Zeitraum von 25 Jahren von 1995 bis 2020 retrospektiv nach Fällen mit Einschränkungen von konjugierten vertikalen Augenbewegungen durchsucht und hinsichtlich ihrer orthoptischen Charakteristiken und zugrunde liegenden Pathologien quantitativ ausgewertet. Ziel der Studie war es, unterschiedliche Ausprägungen der vBP v. a. hinsichtlich langsamer und schneller Augenbewegungen darzustellen.

\section{Resultate}

Es wurden 202 Patienten mit vBP identifiziert, davon waren 118 (58\%) männlich und 84 (42\%) weiblich. Das Alter lag im Durchschnitt bei 44 Jahren mit einer Spannweite von 0 bis 89 Jahren und Häufigkeitsgip- 


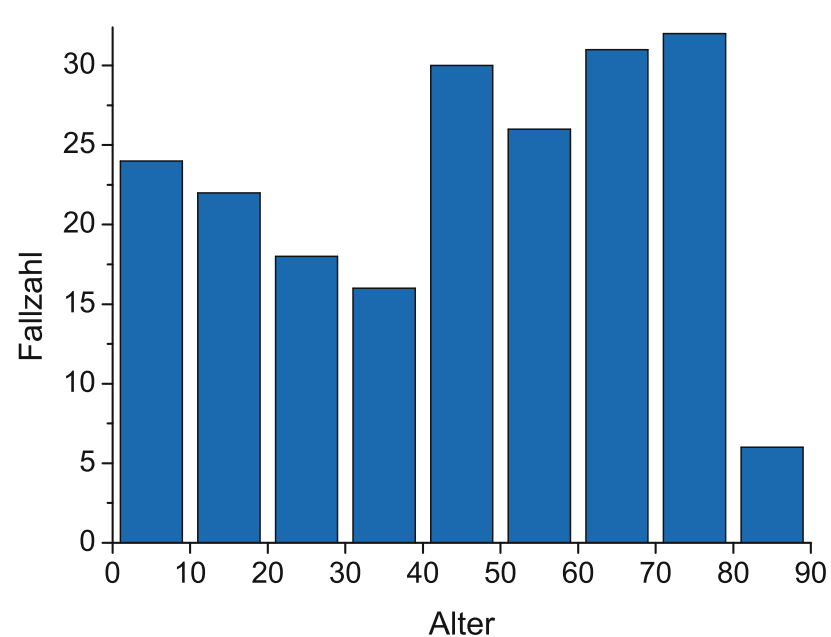

Abb. 2 Altersverteilung der Patienten mit vertikaler Blickparese gruppiert nach Lebensdekade

feln in der ersten, fünften und achten Lebensdekade (Abb. 2).

Es hatten 103 Patienten (51\%) eine vBP mit Einschränkung schneller und langsamer Blickbewegungen nach oben und unten. Bei 31 Patienten waren diese komplett ausgefallen. Die Mehrzahl der übrigen Fälle mit vBP in beide Richtungen zeigte eine überwiegende Einschränkung des Aufblicks. Darunter waren auch 12 Patienten mit Dissoziation unter Einschränkung oder Ausfall nur der Sakkaden in eine Richtung (bei 11 nach unten, bei 1 nach oben). Weitere 47 Patienten $(23 \%)$ hatten eine vBP schneller und langsamer Blickbewegungen nur nach oben und 3 (1\%) nur nach unten. Eine Sakkadenparese unter Erhalt der anderen Blickmodalitäten trat bei 27 Patienten (13\%) in beide Vertikalrichtungen auf und bei 21 Patienten (10\%) nur nach oben. Eine isolierte Sakkadenparese nur nach unten wurde nicht beobachtet. Eine isolierte vBP oh-

\section{Alter $<18$ \\ ( $n=45)$}

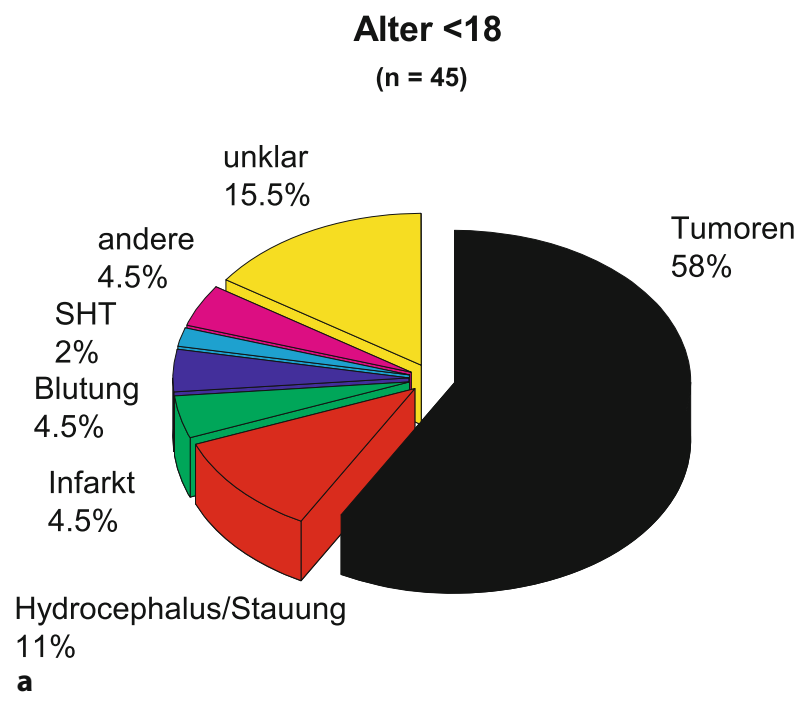

ne weitere Störungen trat bei nur 12 Patienten (6\%) auf.

Die Ätiologien bei Kindern und Jugendlichen bzw. im höheren Erwachsenenalter zeigten sehr unterschiedliche Verteilungen: Bei Patienten bis zum 18. Lebensjahr waren in $58 \%$ der Fälle Tumoren ursächlich (Abb. 3a), während bei Patienten über 60 v. a. degenerative Erkrankungen (44\%) und Infarkte (41\%) vorkamen (Abb. 3b).

\section{Dorsales Mittelhirnsyndrom}

Es wurden 155 Patienten (77\%) auf Grundlage der klinischen und radiologischen Befunde ätiologisch einem dorsalen Mittelhirnsyndrom (DMS) zugeordnet, unter Einschluss von 10 Patienten mit isolierter vBP und 1 Patienten mit asymmetrischer vBP und Konvergenzparese. Bei nur 18 Patienten (12\%) entsprach die Symptomatik dem von Parinaud geprägten Syndrombild (vBP + Konvergenzparese \pm Pupillenstörung ohne weitere Symptome). Der Großteil der Patienten mit DMS (88\%) hatte eine Parese der Vertikalbewegungen nur oder vorwiegend nach oben, während $19 \mathrm{~Pa}-$ tienten (12\%) ein „reverse pattern“ mit überwiegender oder ausschließlicher Parese nach unten zeigten. Die Tab. 1 listet alle beobachteten okulomotorischen Störungen und ihre Häufigkeit auf. Knapp drei Viertel der Fälle $(73 \%)$ hatte eine Einschränkung langsamer und schneller Vertikalbewegungen, wobei die Paresen nach oben in Anzahl und Ausmaß prädominierten. Bei zumindest 7 Patienten war der VOR trotz Parese der willkürlichen Augenbewegungen erhalten. Eine Dissoziation mit Blickfolge- und Sakkadenparese in eine und nur Sakkadenparese in die andere Richtung trat in 8 Fällen auf. Etwas mehr als ein Viertel der Fälle $(27 \%)$ zeigte eine reine Sakkadenparese unter Erhalt der anderen Blickmodalitäten. Auch bei diesen Patienten waren die Aufwärtssakkaden häufiger und

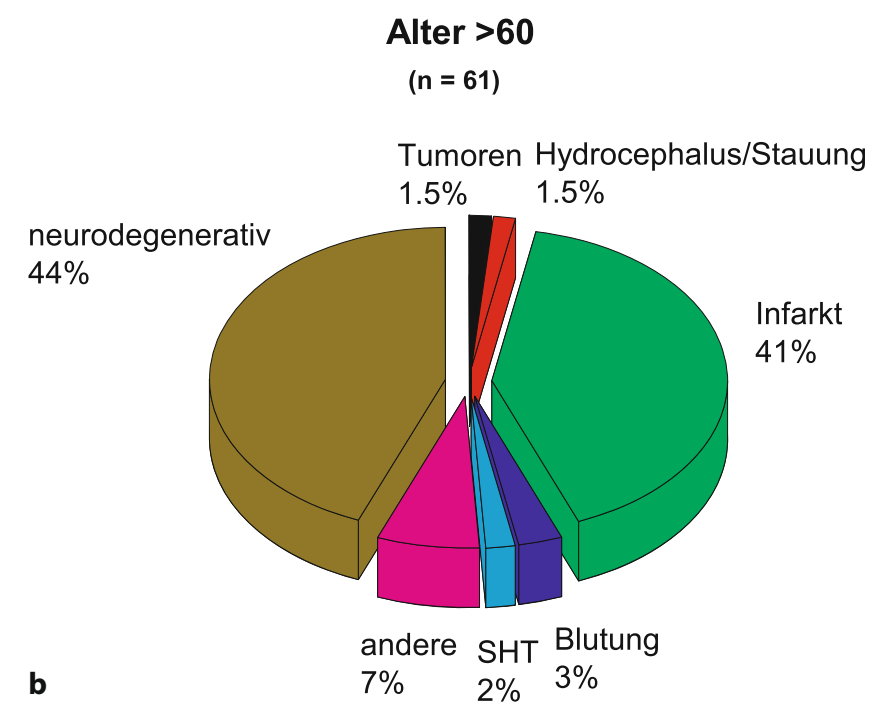

Abb. 3 Häufigkeitsverteilung der Ätiologien vertikaler Blickparesen bei Kindern und Jugendlichen (a) und bei Erwachsenen über 60 Jahren (b). SHT Schädel-Hirn-Trauma 


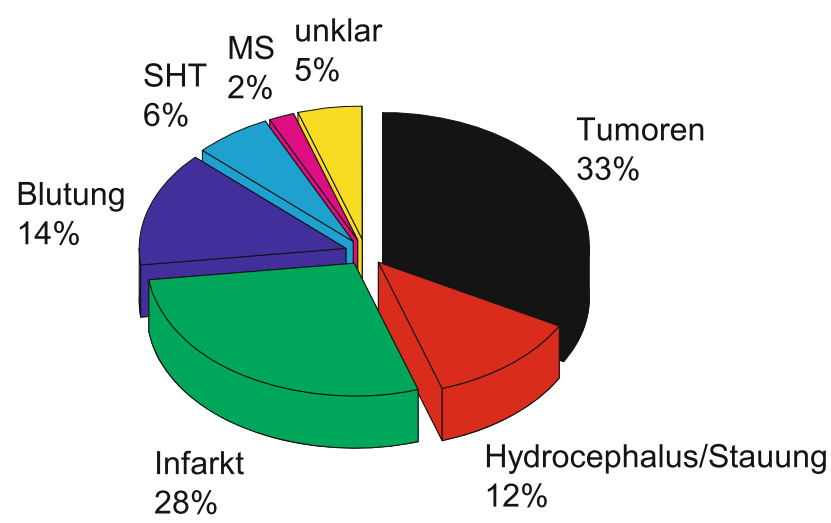

Abb. 4 Häufigkeitsverteilung der Ätiologien bei Patienten mit dorsalem Mittelhirnsyndrom. SHT Schädel-Hirn-Trauma, MS multiple Sklerose

meistens stärker betroffen, jedoch gab es auch 3 Fälle mit einem „reverse pattern“ der Sakkadenparese.

Eine Konvergenzparese war mit $49 \%$ das häufigste Begleitsymptom und war zumeist mit einem Retraktionsnystagmus vergesellschaftet. Als weitere typische Syndromzeichen fanden sich bei knapp einem Drittel der Patienten eine gestörte Lichtreaktion der Pupille (in 13 Fällen mit beschriebener Licht-Nah-Dissoziation), in je einem Fünftel der Fälle eine Skew Deviation oder Ocular Tilt Reaction (OTR) bzw. ein Spontan- oder Blickrichtungsnystagmus sowie in 9 Fällen eine Oberlidretraktion (s Tab. 1). Alle Begleitsymptome kamen in unterschiedlicher Kombination bei allen Ausprägungen der vBP vor. Es zeigten 58 Patienten mit DMS (37\%) weitere Okulomotorikstörungen zentraler Genese, darunter Paresen von N.III, N.IV, N.VI und N.VII oder horizontale Bewegungsstörungen einschließlich Blickparese, Sakkadenstörung, internukleäre Ophthalmoplegie, Esotropie und Exotropie.

Die Ursachen eines DMS waren am häufigsten Tumoren, davon ein Drittel Pinealistumoren, sowie ischämische oder hämorrhagische Insulte im Bereich Thalamus/Mittelhirn (Abb. 4). Weniger häufig waren Hydrozephalus, traumatische Genese und multiple Sklerose.

\section{Neurodegenerative Erkrankungen}

Die 33 Patienten mit vBP durch neurodegenerative Erkrankungen hatten durchschnittlich ein höheres Alter und zeigten in mehr als drei Viertel der Fälle sowohl Einschränkungen vertikaler als auch horizontaler Augenbewegungen, jedoch waren die vertikalen Augenbewegungen in der Regel deutlich stärker betroffen (Tab. 1). Sechs Patienten (18\%) hatten sogar nur eine vBP ohne Störung der horizontalen Bewegungen. In 13 Fällen gab es einen kompletten Ausfall aller vertikalen Augenbewegungen. Bei den übrigen Patienten waren meistens Auf- und Abblick betroffen. In 10 Fällen war die vBP überwiegend oder nur nach oben und in 6 überwiegend nach unten. In 3 Fällen gab es in eine Richtung eine Dissoziation mit Einschränkung le-
Tab. 1 Aufstellung der Charakteristiken von Patienten mit vertikaler Blickparese gruppiert nach Diagnosen und Gegenüberstellung mit einer früheren Fallserie von Patienten mit prätektalem Syndrom [9]

\begin{tabular}{|l|l|l|l|l|} 
& $\begin{array}{l}\text { Dorsales } \\
\text { Mittelhirn- } \\
\text { syndrom }\end{array}$ & $\begin{array}{l}\text { Prätektales } \\
\text { Syndrom }\end{array}$ & $\begin{array}{l}\text { Neurodege- } \\
\text { nerative } \\
\text { Erkrankungen }\end{array}$ & $\begin{array}{l}\text { Mitochon- } \\
\text { driale }\end{array}$ \\
$\begin{array}{l}\text { Erkran- } \\
\text { kungen }\end{array}$
\end{tabular}

diglich der Sakkaden. Auffällig war der hohe Anteil an Patienten mit Konvergenzparese (79\%). Dadurch ergaben sich bei 5 Patienten Befunde wie bei einem „Parinaud-Syndrom“ (vBP + Konvergenzparese). 
Ätiologisch stellten in dieser Gruppe Patienten mit Steele-Richardson-Olszewski-Syndrom (progressive supranukleäre Blickparese [PSP]) den überwiegenden Anteil (28 Patienten). Weitere Diagnosen umfassten 3 Patienten mit klinisch im Vordergrund stehendem Morbus Parkinson und geringer ausgeprägten Okulomotorikstörungen und je 1 Patienten mit spinozerebellärer Ataxie und Lewy-Körperchen-Demenz. Bei den meisten Patienten mit neurodegenerativen Erkrankungen war die Augenbewegungsstörung das Initialsymptom.

\section{Andere Diagnosen}

Seltenere Diagnosen bei vBP umfassten mitochondriale Erkrankungen in 6 Fällen (chronisch progrediente externe Ophthalmoplegie [CPEO], okulopharyngeale Dystrophie), welche teilweise zusätzliche Okulomotorikstörungen zeigten (Tab. 1), sowie benigne Störungen bei 4 Säuglingen ohne zerebrale Pathologie, welche als transientes Sonnenuntergangsphänomen [4] bzw. tonische Blickdeviation nach unten [5] klassifizierbar waren. Auch hier fand sich zum Teil eine Konvergenzminderung, Oberlidretraktion oder ein erhaltener VOR. In der orthoptischen Untersuchung imponierten noch je 1 Patient mit Miller-Fisher-Syndrom bzw. beidseitiger N.III-Parese sowie 2 Patienten ungeklärter Ätiologie mit dem Bild einer vBP.

Längerfristige Verlaufsuntersuchungen waren nur bei 63 der 202 Fälle vorhanden. Davon zeigten $38 \mathrm{~Pa}$ tienten mit DMS sowie jene mit transientem Sonnenuntergangsphänomen und Miller-Fisher-Syndrom eine komplette oder deutliche Besserung.

\section{Schlussfolgerung}

Die vBP ist durch eine Bewegungseinschränkung der konjugierten vertikalen Augenbewegungen nach oben und/oder nach unten charakterisiert, wobei isolierte Paresen nach unten nur selten vorkommen. Ursache sind in der Regel beidseitige und seltener einseitige Läsionen im rostralen Mittelhirn, in erster Linie durch ischämische oder hämorrhagische Insulte, Tumoren mit oder ohne Liquorzirkulationsstörung und Hydrozephalus, aber auch durch entzündliche oder neurodegenerative Erkrankungen, Speicherkrankheiten und Psychopharmaka [1, 2].

Das orthoptische Befundspektrum in unserer Fallserie reichte von einer isolierten Sakkadenstörung nur nach oben bis hin zum kompletten Ausfall aller willkürlichen und reflektorischen vertikalen Augenbewegungen in beide Richtungen. Meistens waren alle Bewegungsmodalitäten in beiden Richtungen betroffen, jedoch lag dabei auch öfter eine Dissoziation vor, wobei die Sakkaden mehr als die langsamen Augenbewegungen und diese mitunter mehr als der VOR eingeschränkt sein können. Diese Hierarchie der Beeinträchtigung wurde schon früh von Bielschowsky bemerkt [6]. Läsionen der PC führen zu vBP aller
Modalitäten, aufgrund der eingangs beschriebenen Faserverläufe vorwiegend im Aufblick, was sich auch in unserer Fallserie mit DMS zeigt. Im Rahmen eines DMS ist die vBP durch Störung nahe gelegener Strukturen häufig mit zusätzlichen Symptomen vergesellschaftet. Bei begleitender Störung der Konvergenzneurone in der SOA ergibt sich das von Parinaud geprägte Syndrombild einer vBP mit Konvergenzparese. Eine Pupillenstörung ist dabei oft vorhanden, aber schon in der Beschreibung von Parinaud nicht obligat [7]. Obwohl Parinaud nicht nur eine vBP nach oben beschrieb, wurde später bei Überwiegen der vBP nach unten mit begleitender Konvergenzparese der Begriff „reverse Parinaud-Syndrom“ geprägt [8]. Durch die Variabilität weiterer Symptome, die bei Läsionen des oberen Mittelhirns gemeinsam mit einer vBP auftreten können, und auch aufgrund der uneinheitlichen Verwendung des Begriffs „ParinaudSyndrom" in der Literatur wird heute eher die Verwendung der allgemeiner gefassten Begriffe „dorsales Mittelhirnsyndrom“ oder "prätektales Syndrom“ empfohlen [9, 10]. Typische weitere Motilitätsstörungen bei DMS sind dabei neben der Konvergenzparese ein Konvergenznystagmus mit oder ohne Bulbusretraktion v. a. im Aufblick, eine verminderte Lichtreaktion der Pupille unter besserer Erhaltung der Konvergenzmiose (Licht-Nah-Dissoziation aufgrund getrennt verlaufender exzitatorischer Fasern von den prätektalen Nuclei), horizontales Schielen (Exotropie durch verminderten oder Esotropie durch erhöhten Konvergenztonus, Konvergenzspasmus, Divergenzparese bzw. Pseudoabduzensparese), Oberlidretraktion (durch Enthemmung des Nucleus caudalis centralis, Collier-Zeichen), Ptosis, Skew Deviation (im Rahmen einer kontraversiven OTR) und vertikaler Spontanoder Blickrichtungsnystagmus. Ophthalmoskopisch können auch noch Stauungspapillen vorliegen, wenn z. B. im Rahmen einer Aquäduktstenose der intrakranielle Druck erhöht ist.

In der bisher größten Fallserie von 206 Patienten mit prätektalem Syndrom zeigten nahezu alle Patienten eine verminderte Lichtreaktion als häufigstes Symptom und $87 \%$ eine vBP ([9]; Tab. 1). Darunter fanden sich anders als in unserer Studie wesentlich mehr Patienten mit einer Blickparese nur nach oben als in beide Richtungen, allerdings hatte dort ein hoher Anteil eine Blickdeviation nach unten. Außerdem wurden in dieser Studie keine Patienten mit alleiniger Sakkadenparese eingeschlossen, da laut dem $\mathrm{Au}-$ tor diese nicht dem prätektalen Syndrom mit Läsion der PC zugeordnet werden könne. Unsere Fallserie zeigt aber, dass typische Symptome, insbesondere die Konvergenzparese, aber auch Pupillenstörung, Konvergenznystagmus, OTR und Lidretraktion auch bei alleiniger vertikaler Sakkadenparese auftreten und somit durchaus im Rahmen eines DMS vorhanden sind. Wie für das DMS typisch fand sich auch bei den alleinigen Sakkadenparesen unserer Patienten bevorzugt eine Störung des Aufblicks. Die eingangs beschriebene 
Verschaltung der Sakkaden im Mittelhirn zeigt insgesamt einen bevorzugten Faseraustausch bei Aufwärtssakkaden, was diese Augenbewegung v. a. bei größeren mittelliniennahen Läsionen empfindlicher gegenüber Störungen machen könnte. Die Konvergenzparese war in unserer Fallserie das häufigste Begleitsymptom und wurde wesentlich öfter beobachtet als bei Keane. Umgekehrt wurden bei unseren Patienten eine Pupillenstörung und Oberlidretraktion wesentlich seltener beobachtet. Es ist nicht auszuschließen, dass diesen Befunden in manchen unserer Fälle zu wenig Beachtung geschenkt wurde. Störungen horizontaler Augenbewegungen waren insgesamt ähnlich häufig, stellten sich bei unseren Patienten aber anders verteilt dar. Weitere Hirnnervenparesen (N.III, N.IV, N.VII) waren nur in unserer Fallserie auffällig. Ansonsten decken sich die Häufigkeiten der einzelnen Symptome unserer Fallserie mit DMS recht gut mit der von Keane.

Frühere Studien zeigen, dass isolierte vertikale Sakkadenparesen bei bilateralen Läsionen des Sakkadengenerators riMLF auftreten, wobei der Aufblick durch die bilaterale Verschaltung weniger stark betroffen sein kann [1, 2]. Aus demselben Grund sind bei den selteneren Läsionen eines Kerns lediglich die Abwärtssakkaden leicht verlangsamt, jedoch tritt dann auch ein kontraversiver torsioneller Nystagmus auf. Isolierte bilaterale Läsionen des INC hingegen führen zu einer Parese aller vertikalen Augenbewegungen, wobei die Sakkadengeschwindigkeit nicht verlangsamt ist. Durch die gestörte Blickhaltefunktion kommt es hier oft zu einem Upbeat-Nystagmus. Einseitige Läsionen führen $\mathrm{zu}$ einem ipsiversiven torsionalen Nystagmus und zu einer kontraversiven OTR [11]. Störungen der vertikalen Augenbewegungen durch solche isolierten Kernläsionen, sind auch in Anbetracht unserer Ergebnisse eher selten, da schädigende Pathologien meistens größere Areale betreffen und Kern- und Interneurone auch nur teilweise geschädigt werden können. Es ist gut nachvollziehbar, dass sich dadurch bei Mittelhirnläsionen verschiedenartige Ausprägungen einer vBP mit diversen Begleitsymptomen ergeben können. So verursachen beispielsweise auch einseitige Mittelhirnläsionen unter Einbeziehung von riMLF und INC aber mit Aussparung der PC eine vBP nach oben oder in beide Richtungen mit oder ohne Dissoziation einzelner Blickmodalitäten [12-17] oder auch ein DMS [18, 19]. Auch einseitige Thalamusläsionen können eine vBP in eine oder beide Richtungen verursachen [20]. Eine vBP nur nach unten wäre auch bei einseitigen Läsionen in Mittelhirn denkbar [21], wurde aber bisher nicht im Zusammenhang mit einem DMS beschrieben. Zwei Patienten unserer Fallserie mit vBP nach unten mit begleitender Konvergenzparese bei einseitigen Läsionen hatten auch diskret hypometrische Sakkaden nach oben. Patienten mit begleitender Abduzensparese oder horizontaler Blickparese hatten zum Teil eine Pseudoabduzensparese bei thalamomesenzephalen
Läsionen [22] oder, wie auch alle Patienten mit Fazialisparese, multiple Läsionen mit Beteiligung der Pons.

In unserer Studie nähern wir uns dem Thema vBP von der klinisch-orthoptischen Seite an, weshalb wir auch Patienten mit anderen Ätiologien als dem DMS analysiert haben. Am häufigsten war darunter die PSP, welche somit eine wichtige Differenzialdiagnose v. a. im höheren Lebensalter darstellt. Hier kommt es durch Anhäufung von tau-Protein im Hirnstammgewebe zum degenerativen Verlust der Neuronen für vertikale und auch horizontale Augenbewegungen und Konvergenz, wobei die Paresen wie auch in unserer Fallserie eher kein Richtungsüberwiegen zeigen [23]. Durch den langsam progredienten Verlauf, die häufig gemeinsam auftretenden horizontalen Bewegungsstörungen und das Fehlen anderer zentraler Okulomotorikstörungen ist die PSP in der Regel klinisch gut differenzierbar. Der VOR ist hier charakteristischerweise oft noch besser erhalten. Bis auf Letzteres imponieren die Motilitätsstörungen bei Mitochondriopathien recht ähnlich. Beim akuten MillerFisher-Syndrom sind differenzialdiagnostisch die serologischen Zusatzbefunde wegweisend. Eine rasche radiologische Abklärung von zerebralen Pathologien ist beim erstmaligen Auftreten einer vBP allerdings in jedem Fall angebracht.

Zusammenfassend verdeutlichen unsere Ergebnisse, dass langsame und schnelle Augenbewegungen nach oben und unten aufgrund unterschiedlicher Organisation im Mittelhirn bei dortigen Pathologien verschieden stark gestört sein können, und veranschaulichen die enge Lagebeziehung der Zentren für vertikale Augenbewegungen, Konvergenz und Pupillenreaktion im dorsalen Mittelhirn.

Funding Open access funding provided by Medical University of Vienna.

\section{Einhaltung ethischer Richtlinien}

Interessenkonflikt B. Pemp, S. Koinig, K. Kircher, C. Mitsch und A. Reitner geben an, dass kein Interessenkonflikt besteht.

Ethische Standards Alle im vorliegenden Manuskript beschriebenen Untersuchungen wurden von der zuständigen Ethikkommission zugelassen und im Einklang mit den ethischen Standards der Deklaration von Helsinki durchgeführt.

Open Access Dieser Artikel wird unter der Creative Commons Namensnennung 4.0 International Lizenz veröffentlicht, welche die Nutzung, Vervielfältigung, Bearbeitung, Verbreitung und Wiedergabe in jeglichem Medium und Format erlaubt, sofern Sie den/die ursprünglichen Autor(en) und die Quelle ordnungsgemäß nennen, einen Link zur Creative Commons Lizenz beifügen und angeben, ob Änderungen vorgenommen wurden.

Die in diesem Artikel enthaltenen Bilder und sonstiges Drittmaterial unterliegen ebenfalls der genannten Creative Commons Lizenz, sofern sich aus der Abbildungslegende nichts anderes ergibt. Sofern das betreffende Material nicht unter der genannten Creative Commons Lizenz steht und die be- 
treffende Handlung nicht nach gesetzlichen Vorschriften erlaubt ist, ist für die oben aufgeführten Weiterverwendungen des Materials die Einwilligung des jeweiligen Rechteinhabers einzuholen.

Weitere Details zur Lizenz entnehmen Sie bitte der Lizenzinformation auf http://creativecommons.org/licenses/by/4. 0/deed.de.

\section{Literatur}

1. Wong AMF. Eye movement disorders. New York: Oxford University Press; 2008.

2. Leigh RJ, Zee DS. The neurology of eye movements. New York: Oxford University Press; 2015.

3. May PJ, Warren S, Gamlin PDR, Billig I. An anatomic characterization of the midbrain near response neurons in the macaque monkey. Invest Ophthalmol Vis Sci. 2018;59:1486-502.https://doi.org/10.1167/iovs.17-23737.

4. Cernerud L. The setting-sun eye phenomenon in infancy. Dev Med Child Neurol. 1975;17:447-55. https://doi.org/10. 1111/j.1469-8749.1975.tb03496.x.

5. Hoyt CS, Mousel DK, Weber AA. Transient supranuclear disturbances of gaze in healthy neonates. Am J Ophthalmol. 1980;89:708-13. https://doi.org/10.1016/00029394(80)90292-5.

6. Bielschowsky A. Lectures on motor anomalies of the eyes: III. Paralyses of the conjugate movements of the eyes. Arch Ophthalmol. 1935;13:569-83. https://doi.org/ 10.1001/archopht.1935.00840040057003.

7. Parinaud MH. Paralysie des mouvements associès desyeux. Arch Neurol. 1883;5:145-72.

8. Cogan DC. Paralysis of down-gaze. Arch Ophthalmol. 1974;91:192-9. https://doi.org/10.1001/archopht.1974. 03900060200009.

9. Keane JR. The pretectal syndrome: 206 patients. Neurology. 1990;40:684-90.https://doi.org/10.1212/wnl.40.4.684.

10. Thömke F. Kapitel 4.3.1, Dorsales Mittelhirnsyndrom. In: Augenbewegungsstörungen. 3. Aufl. Bad Honeff: Hippocampus;2016. S. 200-1.

11. Brandt T, Dieterich M. Two types of ocular tilt reaction: the 'ascending' pontomedullary VOR-OTR and the 'descending' mesencephalic integrator-OTR. Neuroophthalmology. 1998;19:83-92. https://doi.org/10.1076/noph.19. 2.83.3944.

12. Ranalli PJ, Sharpe JA, Fletcher WA. Palsy of upward and downward saccadic, pursuit, and vestibular movements with a unilateral midbrain lesion: pathophysiologic correlations. Neurology. 1988;38:114-22. https://doi.org/10. 1212/wnl.38.1.114.
13. Bogousslavsky J, Miklossy J, Regli F, Janzer R. Vertical gaze palsy and selective unilateral infarction of the rostral interstitial nucleus of the medial longitudinal fasciculus (riMLF). J Neurol Neurosurg Psychiatry. 1990;53:67-71. https://doi. org/10.1136/jnnp.53.1.67.

14. Hommel M, Bogousslavsky J. The spectrum of vertical gaze palsy following unilateral brainstem stroke. Neurology. 1991;41:1229-34.https://doi.org/10.1212/wnl.41.8.1229.

15. Sharpe JA, Kim JS. Midbrain disorders of vertical gaze: a quantitative re-evaluation. Ann N Y Acad Sci. 2002;956:143-54. https://doi.org/10.1111/j.1749-6632. 2002.tb02815.x.

16. Alemdar M, Kamaci S, Budak F. Unilateral midbrain infarction causing upward and downward gaze palsy. J Neuroophthalmol. 2006;26:173-6. https://doi.org/10.1097/01. wno.0000235588.18169.de.

17. Kang JH, Sharpe JA. Dissociated palsy of vertical saccades: loss of voluntary and visually guided saccades with preservation of reflexive vestibular quick phases. J Neuroophthalmol. 2008;28:97-103. https://doi.org/10.1097/wno. ob013e3181772647.

18. Moriyasu H, Hashimoto Y, Miyashita T, Satomi M, Yamaguchi T. Supranuclear vertical gaze palsy and convergence nystagmuscaused byunilateral riMLFlesion. RinshoShinkeigaku. 1991;31:1235-7.

19. Serino J, Martins J, Páris L, Duarte A, Ribeiro I. Parinaud's syndrome due to an unilateral vascular ischemic lesion. Int Ophthalmol. 2015;35:275-9. https://doi.org/10.1007/ s10792-015-0045-y.

20. Moon Y, Eah KS, Lee EJ, et al. Neuro-ophthalmologic features and outcomes of thalamic infarction: a single-institutional 10-year experience. J Neuroophthalmol. 2019; https://doi.org/10.1097/wno.0000000000000864.

21. Iwasaki Y, Matsuda K, Sugasawa J, Utsumi T. A case of supranuclear downward gaze paralysis due to infarction at the rostral interstitial MLF detected by MRI. Shinkei Ganka. 1987;4:206-10.

22. Pullicino P, LincoffN, TruaxBT.Abnormalvergence with upper brainstem infarcts: pseudoabducens palsy. Neurology. 2000;55:352-8. https://doi.org/10.1212/wnl.55.3.352.

23. Chen AL, Riley DE, King SA, et al. The disturbance of gaze in progressive supranuclear palsy: implications for pathogenesis. Front Neurol. 2010;1:147. https://doi.org/ 10.3389/fneur.2010.00147.

Hinweis des Verlags Der Verlag bleibt in Hinblick auf geografische Zuordnungen und Gebietsbezeichnungen in veröffentlichten Karten und Institutsadressen neutral. 